\title{
Microarray analysis of circular RNA expression patterns in polarized macrophages
}

\author{
YINGYING ZHANG ${ }^{1^{*}}$, YAO ZHANG ${ }^{2 *}$, XUEQIN LI ${ }^{3 *}$, MENGYING ZHANG $^{3}$ and $\mathrm{KUN} \mathrm{LV}^{3}$ \\ ${ }^{1}$ Laboratory Medicine, Yijishan Hospital, Wannan Medical College; ${ }^{2}$ Department of Biochemistry, Wannan Medical College; \\ ${ }^{3}$ Central Laboratory, Yijishan Hospital, Wannan Medical College, Wuhu, Anhui 241001, P.R. China
}

Received November 30, 2015; Accepted January 5, 2017

DOI: $10.3892 /$ ijmm.2017.2852

\begin{abstract}
Circular RNAs (circRNAs) are generated from diverse genomic locations and are a new player in the regulation of post-transcriptional gene expression. Recent studies have revealed that circRNAs play a crucial role in fine-tuning the level of microRNA (miRNA)-mediated regulation of gene expression by sequestering miRNAs. The interaction of circRNAs with disease-associated miRNAs suggests that circRNAs are important in the pathology of disease. However, the effects and roles of circRNAs in macrophage polarization have yet to be explored. In the present study, we performed a circRNA microarray to compare the circRNA expression profiles of bone marrow-derived macrophages (BMDMs) under two distinct polarizing conditions (M1 macrophages induced by interferon- $\gamma$ and LPS stimulation, and M2 macrophages induced by interleukin-4 stimulation). Our results showed that a total of 189 circRNAs were differentially expressed between M1 and M2 macrophages. Differentially expressed circRNAs with a high fold-change were selected for validation by RT-qPCR: circRNA-003780, circRNA-010056, and circRNA-010231 were upregulated and circRNA-003424, circRNA-013630, circRNA-001489 and circRNA-018127 were downregulated (fold-change $>4, \mathrm{P}<0.05$ ) in $\mathrm{M} 1$ compared to M2, which was found to correlate with the microarray data. Furthermore, the most differentially expressed circRNAs within all the comparisons were annotated in detail with circRNA/miRNA interaction information using miRNA target prediction software. In conclusion, the present study provides novel insight into the role of circRNAs in macrophage differentiation and polarization.
\end{abstract}

Correspondence to: Dr Kun Lv, Central Laboratory, Yijishan Hospital, Wannan Medical College, 2 Western Zheshan Road, Wuhu, Anhui 241001, P.R. China

E-mail: lvkun315@126.com

*Contributed equally

Key words: polarization, bone marrow-derived macrophages, circular RNA, miRNA, microarray

\section{Introduction}

Macrophages are an important component of innate immunity, and play a major function in host homeostasis (1). Macrophages are versatile multifunctional cells, which change their phenotype and functional capacity depending on various conditions. In response to micro-environmental signals, macrophages undergo differential activation, including classical activation characterized by a pro-inflammatory phenotype (also called M1), and alternative activation (M2) characterized by polarization associated with an anti-inflammatory profile (2). Flexibility and plasticity are key features of mononuclear phagocytes, and specific cytokine stimuli can induce mononuclear phagocytes to express specialized and polarized functional properties $(3,4)$. Classical polarized M1 macrophages activated by interferon- $\gamma($ IFN $-\gamma)$ or other microbial products such as LPS, in turn produce high levels of pro-inflammatory cytokines, such as tumor necrosis factor- $\alpha$ (TNF- $\alpha$ ), interleukin (IL)-12, IL-23, IL-6, IL-1 $\beta$ and a high production of reactive oxygen and nitrogen intermediates. M1 macrophages contribute as inducer and effector cells in promotion of Th1 responses, and mediate resistance against intracellular parasites and tumor cells (5-9). In contrast, alternatively activated M2 macrophages induced by IL-4, IL-13 or immune complex $(10,11)$, are characterized by a phenotype of low production of IL-12 and IL-13, and high levels of IL-10, Arg-1, YM-1, Fizz1 and Mrc-1 (12). However, the mechanisms regulating the expression of the constellation of genes in the response of macrophages to polarizing conditions remain to be understood.

Circular RNA (circRNAs), formed by the covalent linkage of the ends of a single RNA molecule, are new players in the regulation of post-transcriptional gene expression. circRNAs have been shown to cause loss of microRNA (miRNA) function accompanied by increased levels of endogenous targets, acting as miRNA sponges $(13,14)$. Although circRNAs have been identified for decades now, only recently have studies revealed the numbers of circRNAs that are endogenous to mammalian cells. Many circRNAs are abundant, stable, conserved and potentially function as competing endogenous RNAs (15-17). circRNAs can arise from exons (exonic circRNA) or introns (intronic circRNA), showing distinct modes of generation. Evidence of potential functions in the regulation of gene expression is emerging for both exonic and 
intronic circRNAs $(15,17-20)$. However, the role of circRNAs in gene expression occurring during macrophage polarization remains unknown.

In the present study, we used a specific circRNA microarray platform to investigate the changes in the abundance of circRNAs induced by the activation of primary bone marrow-derived macrophages (BMDMs) under two distinct polarizing conditions to expand the spectrum of the activation patterns of M1 and M2 macrophages. Our data revealed that a number of circRNAs were consistently altered under distinct polarizing conditions. circRNA-miRNA interaction during macrophage polarization was then predicted. Thus, the present study is valuable for further investigation of the precise roles of circRNAs in macrophage differentiation and polarized activation processes.

\section{Materials and methods}

Mice. A total of $40 \mathrm{BALB} / \mathrm{c}$ male mice (8 weeks old) were obtained from the Experimental Animal Center of Qinglongshan (Nanjing, China), and were housed in pathogen-free mouse colonies. All animal experiments were performed according to the guidelines for the Care and Use of Laboratory Animals (Ministry of Health, China, 1998). All experimental protocols were approved by the Animal Ethics Committee of Yijishan Hospital (Wuhan, China).

Isolation and cultivation of murine BMDMs. The mice were sacrificed by cervical dislocation following anesthesia, and the abdomen and hind legs were sterilized with $75 \%$ ethanol. An incision was made in the midline of the abdomen, and clipped outward to expose the hind legs. Scissors were then used to remove all muscle tissue from the bones and the bones were cut at both ends to free them. The bones were crushed in a mortar with $5 \mathrm{ml}$ DMEM. The femur and tibia were separated by cutting at the knee joint. BMDMs were isolated from BALB/c mice by flushing the femurs with Dulbecco's modified Eagle's medium (DMEM) (Gibco, Eggenstein, Germany). The cells were collected in 15-ml tubes and centrifuged for $10 \mathrm{~min}$ at $100 \mathrm{x} \mathrm{g}$. The supernatant was removed, and the pellet was suspended in DMEM containing 20\% fetal bovine serum (FBS) and 20\% L929 supernatant. The cells were then seeded at $1 \times 10^{6}$ cells/well to 6 -well cell-culture plates (Corning Costar, Corning, $\mathrm{NY}, \mathrm{USA}$ ) at $37^{\circ} \mathrm{C}$ in $5 \% \mathrm{CO}_{2}$. After 7 days in culture, the medium was removed and the cells were cultured in RPMI-1640 medium supplemented with 10\% FBS for an additional $24 \mathrm{~h}$. Macrophage polarization was obtained by adding $100 \mathrm{ng} / \mathrm{ml}$ LPS plus $20 \mathrm{ng} / \mathrm{ml} \mathrm{IFN-} \gamma$ (for M1 polarization), or $20 \mathrm{ng} / \mathrm{ml} \mathrm{IL-4} \mathrm{(for} \mathrm{M2} \mathrm{polarization)} \mathrm{in} \mathrm{DMEM}$ containing 10\% FBS and incubating for $48 \mathrm{~h}$.

FACS analysis. BMDMs were harvested and stained with FITC-anti-F4/80 monoclonal antibody (Cat. no. 11-4801-82; eBioscience, Inc., San Diego, CA, USA). Their F4/80 surface expression was assessed by fluorescence-activated cell sorting (FACS) analysis and data were analyzed using CellQuest software (BD Biosciences, Franklin Lakes, NJ, USA).

circRNA microarray analysis. BMDMs were cultured with polarizing stimuli for $48 \mathrm{~h}$; total RNA was extracted by
TRIzol reagent (Invitrogen, Carlsbad, CA, USA) and quantified using the NanoDrop ND-1000. Sample preparation and microarray hybridization were performed based on the Arraystar's standard protocols. Briefly, total RNA from each sample was treated with RNase R (Epicentre Inc., Madison, WI, USA) to remove linear RNAs. Then, each sample was amplified and transcribed into fluorescent cRNA utilizing random primers according to Arraystar's Super RNA Labeling protocol (Arraystar Inc., Rockville, MD, USA). The labeled cRNAs were purified using the RNeasy Mini kit (Qiagen, Hilden, Germany). The concentration and specific activity of the labeled cRNAs (pmol Cy3/ $\mu \mathrm{g}$ cRNA) were measured by NanoDrop ND-1000. Next, $1 \mu \mathrm{g}$ of each labeled cRNA was fragmented by adding $5 \mu \mathrm{l} 10 \mathrm{X}$ blocking agent and $1 \mu \mathrm{l}$ of $25 \mathrm{X}$ fragmentation buffer, and then heating the mixture at $60^{\circ} \mathrm{C}$ for $30 \mathrm{~min}$. Finally, $25 \mu \mathrm{l} 2 \mathrm{X}$ hybridization buffer was added to dilute the labeled cRNA. Subsequently, $50 \mu 1$ of hybridization solution was dispensed into the gasket slide and assembled to the circRNA expression microarray slide. The slides were incubated for $17 \mathrm{~h}$ at $65^{\circ} \mathrm{C}$ in an Agilent hybridization oven. After washing, the slides were scanned by the Axon GenePix 4000B microarray scanner. Scanned images were then imported into GenePix Pro 6.0 software (Axon, Foster City, CA, USA) for grid alignment and data extraction. Quantile normalization and subsequent data processing were performed using the R software package. Differentially expressed circRNAs with statistical significance between the two groups were identified through volcano plot filtering and fold-change filtering. Hierarchical clustering was performed to show the distinguishable circRNA expression pattern among samples. All the experimental results were saved as Microsoft Excel files.

Reverse transcription-quantitative polymerase chain reaction $(R T-q P C R)$. RNA was isolated from the BMDMs as described above. For linear RNA expression analysis, total RNA was reverse transcribed using the SuperScript III First-Strand synthesis system (Life Technologies, Carlsbad, CA, USA) with oligo(dT) according to the manufacturer's instructions. Quantitative PCR (qPCR) was performed using QuantiTect SYBR ${ }^{\circledR}$-Green PCR kits (Qiagen) and glyceraldehyde 3-phosphate dehydrogenase $(G A P D H)$ as an internal control utilizing a Bio-Rad CFX96 real-time PCR system. The reactions were incubated in 96 -well plates at $95^{\circ} \mathrm{C}$ for $5 \mathrm{~min}$, followed by 40 cycles of $95^{\circ} \mathrm{C}$ for $15 \mathrm{sec}$ and $60^{\circ} \mathrm{C}$ for 1 min, followed by a dissociation curve. For circRNA analysis, total RNA was treated with RNase R for enzymatic digestion to remove linear RNAs, and SuperScript III reverse transcriptase using random primers (Life Technologies, Carlsbad, CA, USA) according to the manufacturer's instructions. Primers assaying for circular products were designed to cross the backsplice junction site. qPCR was carried out on a BioRad CFX96 cycler using QuantiTect SYBR ${ }^{\circledR}$-Green PCR kits (Qiagen). All the PCR reactions were run in triplicate. A complete list of primers used in this study is listed in Table I.

Statistical analysis. Data are presented as the means \pm SEM. Statistical analysis of the data was performed with the twotailed independent Student's t-test or ANOVA analysis using GraphPad Prism (version 4.0) statistical program. A value of 
Table I. Primer sequences used in real-time PCR are listed in the 5'-3' direction.

\begin{tabular}{|c|c|}
\hline Genes & Primer $\left(5^{\prime}-3^{\prime}\right)$ \\
\hline Nos 2 & $\begin{array}{l}\text { ATCTTTGCCACCAAGATGGCCTGG } \\
\text { TTCCTGTGCTGTGCTACAGTTCCG }\end{array}$ \\
\hline$T N F-\alpha$ & $\begin{array}{l}\text { CCAGTGTGGGAAGCTGTCTT } \\
\text { AAGCAAAAGAGGAGGCAACA }\end{array}$ \\
\hline$I L-12$ & $\begin{array}{l}\text { GATGTCACCTGCCCAACTG } \\
\text { TGGTTTGATGATGTCCCTGA }\end{array}$ \\
\hline Arg-1 & $\begin{array}{l}\text { TGACTGAAGTAGACAAGCTGGGGAT } \\
\text { CGACATCAAAGCTCAGGTGAATCGG }\end{array}$ \\
\hline$Y M-1$ & $\begin{array}{l}\text { ATGAAGCATTGAATGGTCTGAAAG } \\
\text { TGAATATCTGACGGTTCTGAGGAG }\end{array}$ \\
\hline Fizzl & $\begin{array}{l}\text { AGGTCAAGGAACTTCTTGCCAATCC } \\
\text { AAGCACACCCAGTAGCAGTCATCCC }\end{array}$ \\
\hline$M r c-1$ & $\begin{array}{l}\text { GGAGTGGCAGGTGGCTTAT } \\
\text { TGGACATTTGGGTTCAGGAG }\end{array}$ \\
\hline circRNA-003780 & $\begin{array}{l}\text { AGTGCCTCAGGTTTCTGG } \\
\text { ATTCTGTCTTCCTTTCTTGC }\end{array}$ \\
\hline circRNA-010056 & $\begin{array}{l}\text { TCACCAGGAGAATCCCAGTC } \\
\text { GAACTCTAAAATCAGGCT }\end{array}$ \\
\hline circRNA-010231 & $\begin{array}{l}\text { TTGAGGCGAATGGCTGAG } \\
\text { GCGGGAGGCTTGAATGTC }\end{array}$ \\
\hline circRNA-003424 & $\begin{array}{l}\text { GAAAGCAGCACAGAAATCA } \\
\text { GACCGTGTACTTGCAGACA }\end{array}$ \\
\hline circRNA-013630 & $\begin{array}{l}\text { TAGGTGCTCTAATGTGAGTC } \\
\text { AAAGTTTGGATGAGGGAT }\end{array}$ \\
\hline circRNA-001489 & $\begin{array}{l}\text { GGAAAGGGTCTTAGCAAG } \\
\text { CCCTAAATTACAGCAGGTA }\end{array}$ \\
\hline circRNA-018127 & $\begin{array}{l}\text { AAGACGCTGGCATCCAAA } \\
\text { CCTCGGCTGGCTTCAACA }\end{array}$ \\
\hline
\end{tabular}

TNF- $\alpha$, tumor necrosis factor- $\alpha$; IL-12, interleukin-12.

$\mathrm{P}<0.05$ was considered to indicate a statistically significant difference.

\section{Results}

Identification of ex vivo polarized M1 and M2 macrophages. To identify alterations in the circRNA expression profile during macrophage polarization, we first prepared M1 and M2 phenotype macrophages in vitro. BMDMs were isolated from BALB/c mice and stimulated with LPS and IFN- $\gamma$ (for M1 polarization) or IL-4 (for M2 polarization). FACS analysis showed that the purity of isolated BMDMs was $\sim 96 \%$ by assessing surface F4/80 expression (Fig. 1A). TNF- $\alpha$, Nos2 and IL-12 were used as markers for the M1 phenotype, and other four markers, Arg-1, YM-1, Fizz1 and Mrc-1, were used as markers for the M2 phenotype. BMDMs stimulated with
Table II. The number of differentially expressed circRNAs in the polarized macrophages (M1 vs. M2, expression fold >2).

\begin{tabular}{lcc}
\hline Regulation & Expression fold $>2$ & Expression fold $>4$ \\
\hline Upregulation & 62 & 7 \\
Downregulation & 127 & 27 \\
\hline
\end{tabular}

LPS and IFN- $\gamma$ had significantly higher TNF- $\alpha$, Nos 2 and IL-12 levels, while the levels of Arg1, YM-1, Fizz1 and Mrc-1 were upregulated in IL-4-treated BMDMs (Fig. 1B). These data confirmed that the polarization used in this study resulted in distinct macrophage phenotypes.

Analysis of the circRNA microarray results. To screen for circRNAs that were differentially expressed between the M1 and M2 macrophages, we determined the circRNA expression profiles with a mouse circRNA microarray, and the circRNA expression patterns for M1 and M2 were compared. We found that 189 circRNAs were differentially expressed through a combination of statistical significance (fold-change $>2$; $\mathrm{P}<0.05)$. Among these, 62 circRNAs were upregulated and 127 circRNAs were downregulated in M1 compared with that noted in the M2 macrophages (Table II). The expression ratios $\left(\log _{2}\right.$ scale) of the circRNAs between M1 and M2 are shown as volcano plots at different P-values and fold-change (Fig. 2A) and heat maps (Fig. 2B).

$R T-q P C R$ validation of the differentially expressed circRNAs. To verify the microarray results, we selected 7 differentially expressed exonic circRNAs (fold-change $>4 ; \mathrm{P}<0.05$ ), including 3 upregulated circRNAs and 4 downregulated circRNAs as having the highest fold-change among the differentially expressed circRNAs in M1 compared to M2 by the microarray results, and validated their expression levels by RT-qPCR analysis. The results showed that 3 circRNAs (circRNA-003780, circRNA-010056 and circRNA-010231) were overexpressed, while 4 circRNAs (circRNA-003424, circRNA-013630, circRNA-001489 and circRNA-018127) were underexpressed in M1 compared with M2. The data from RT-qPCR were consistent with the microarray analysis (Fig. 2C).

Annotation for circRNA/microRNA interaction. To further facilitate the implication of our research study, we used the Arraystar's home-made miRNA target prediction software based on TargetScan (21) and miRanda (22) to predict circRNA/microRNA interaction. We selected 29 differentially expressed exonic circRNA with the highest fold-change (fold-change $>4 ; \mathrm{P}<0.05$ ) to predict their microRNA response elements (MREs), including 7 upregulated exonic circRNAs and 22 downregulated circRNAs. Five MREs with good mirSVR scores for each circRNA are shown (Table III). Furthermore, the overexpressed circRNA-010231 (foldchange, 5.56; $\mathrm{P}<0.05)$ in $\mathrm{M} 1$ compared to $\mathrm{M} 2$, showed detailed annotation for interaction with various miRNAs (miR-141-5p, miR-145a-5p, miR-1964-5p, miR-19b-2-5p and miR-6950-5p) (Fig. 3). Moreover, the binding sites of the conserved miRNAs are represented (Fig. 3). 
A

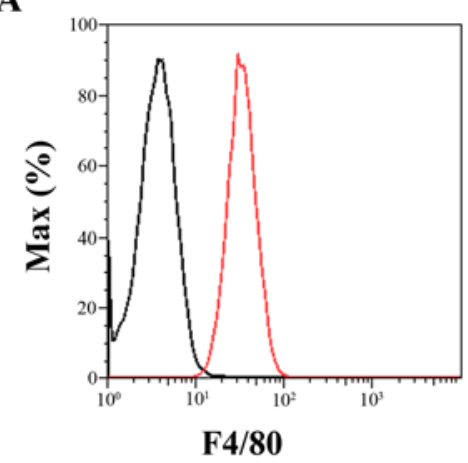

B

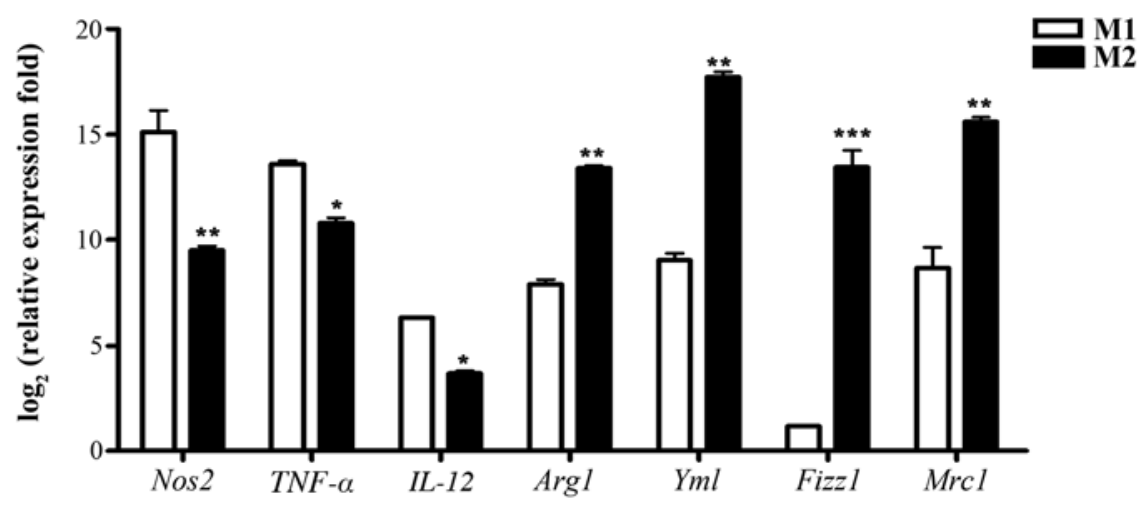

Figure 1. Identification of ex vivo polarized M1 and M2 macrophages. Bone marrow-derived macrophages (BMDM) were isolated from BALB/c mice and treated with LPS (100 ng/ml) and interferon- $\gamma(\mathrm{IFN}-\gamma)(20 \mathrm{ng} / \mathrm{ml})$ for M1 polarization or interleukin-4 (IL-4) (20 ng/ml) for M2 polarization. (A) F4/80 expression was evaluated by FACS analysis. (B) mRNA expression levels of M1 markers Nos2, TNF- $\alpha$ and IL-12; and M2 markers Arg-1, Ym-1 (Chi3l3), Fizz1 (Retnla) and Mrc-1 (CD206) were quantified by RT-q PCR. The data are expressed as the means \pm SEM of three independent experiments.

A

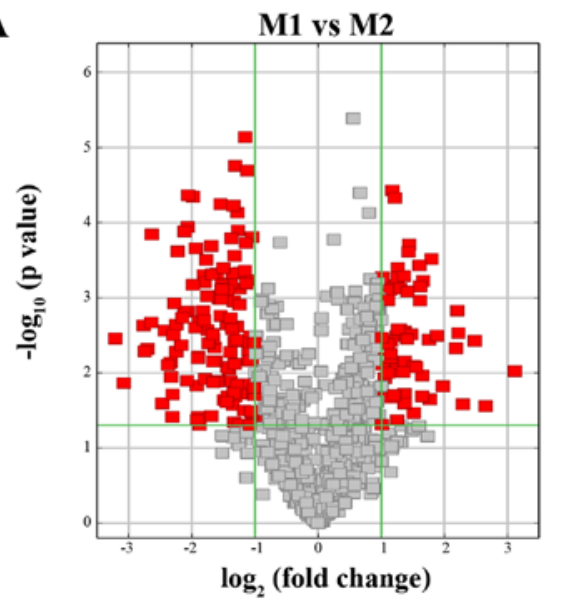

C

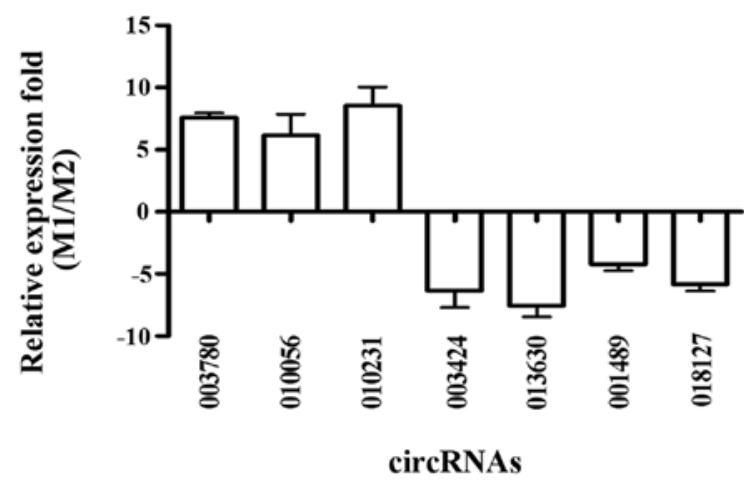

B

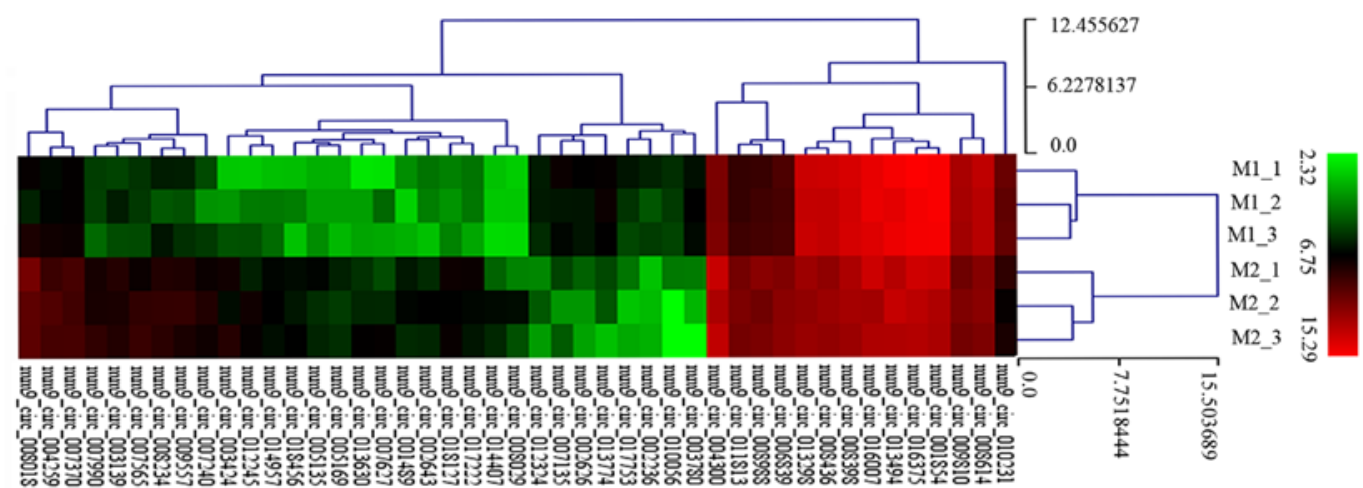

Figure 2. Circular RNA (circRNA) microarray analysis of polarized macrophages. Bone marrow-derived macrophages (BMDMs) were isolated from BALB/c mice and cultured in the presence of LPS (100 ng/ml) plus interferon- $\gamma$ (IFN- $\gamma)(20 \mathrm{ng} / \mathrm{ml})$ or interleukin-4 (IL-4) (20 ng/ml). circRNA microarray was performed to analyze differential circRNA expression in distinct polarized macrophages. (A) Volcano plots comparing the expression of circRNAs in M1 macrophages to M2 macrophages. [Plot of circRNA expression $\log _{2}$-transformed fold-changes (x-axis) vs. - $\log _{10} \mathrm{P}$-value (y-axis)]. The red dots represent the circRNAs having fold-changes $>2.0$ and P-values $<0.05$ between the two types of macrophages; P-value was calculated using the paired t-test. (B) Heat maps of circRNA expression fold-change discriminating M1 macrophages from M2 macrophages. Red indicates a higher fold-change and green indicates a smaller fold-change. The columns represent cirRNAs in the two groups of macrophages while the rows are the significant fold-change of the circRNAs. (C) Confirmation of the differential expression of circRNAs by RT-qPCR. Seven differentially expressed circRNAs were validated by RT-qPCR. The y-axis represents the $\log _{2}$-transformed median fold-change in expression. Data are expressed as the means \pm SEM of three independent experiments.

\section{Discussion}

Mammalian macrophages are induced to diverse phenotypes in response to different external stimuli. We and other researchers have reported that a subset of miRNA expression changes was repeatedly found to be involved in macrophage polarization $(5,6,9,12,23-25)$.

circRNAs, as miRNA sponges, are stable transcripts expressed from diverse genomic locations, and have been recently identified as important players in the regulation of 


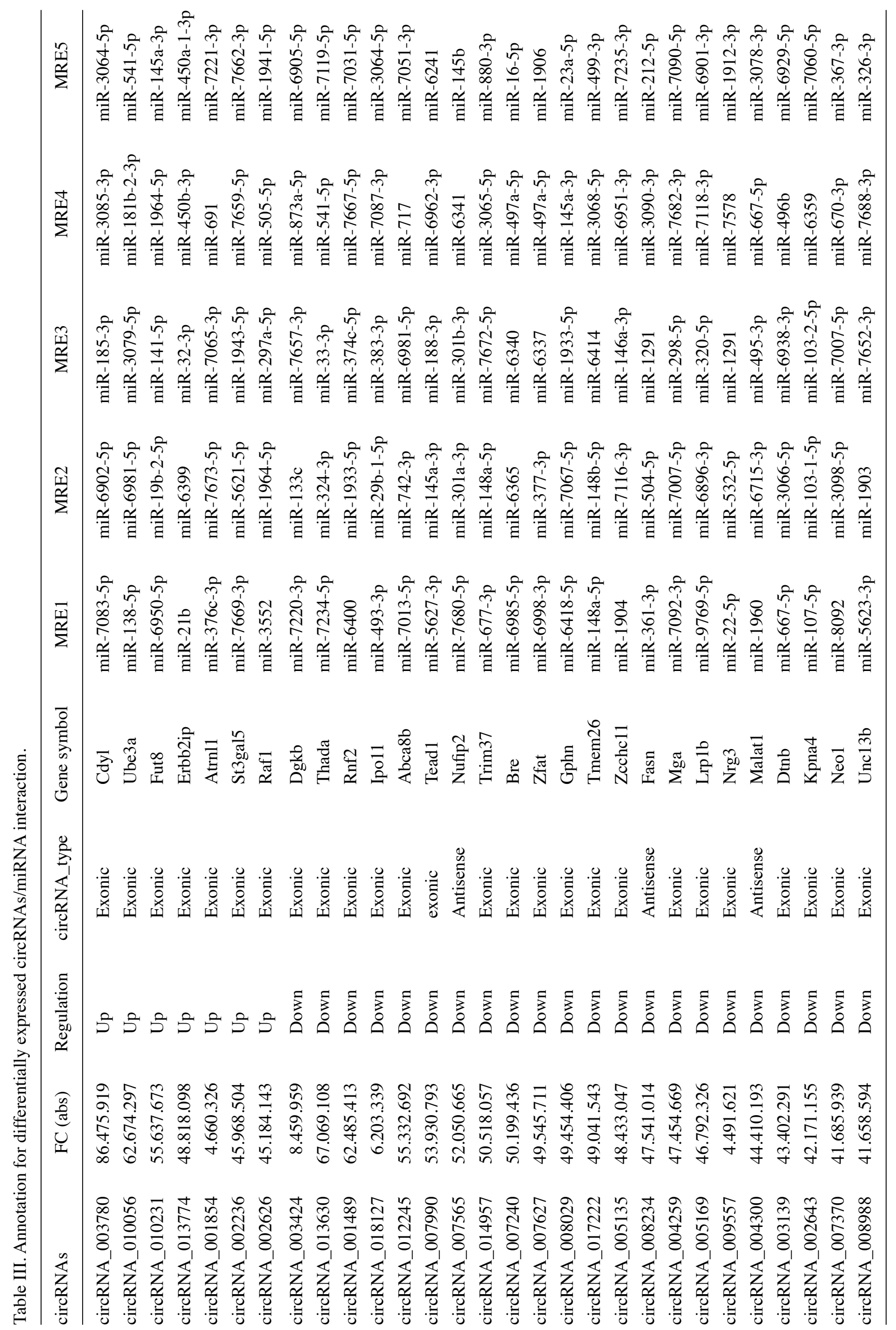




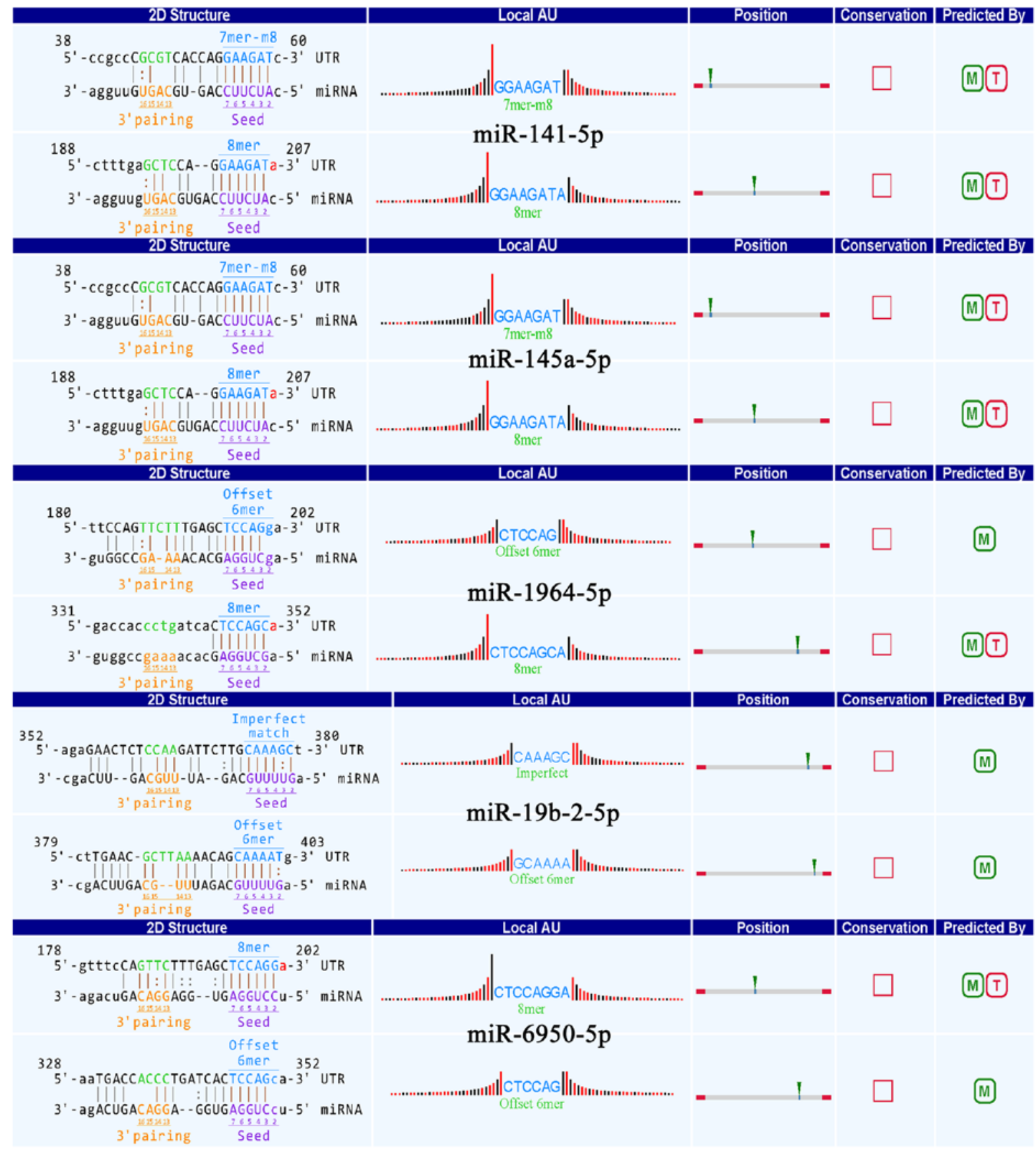

Figure 3. A snippet of the detailed annotation for circRNA-010231/miRNA interaction. The circRNA/miRNA interaction was predicted with Arraystar's home-made miRNA target prediction software based on TargetScan and miRanda, and the most differentially expressed circRNAs within all the comparisons were annotated in detail with the circRNA/miRNA interaction information. Binding sites of conserved miRNAs with good mirSVR scores are represented.

cellular miRNA abundance and thus are a major component in the miRNA-mediated post-transcriptional regulatory network. Available studies suggest that interactions between circRNAs and miRNAs indicate that circRNAs are potentially associated with many disease, cell processes and gene expression $(13,26)$.

The present study aimed to identify the expression patterns of circRNAs in response to stimuli polarizing two distinct patterns of macrophage activation (M1 and M2). We performed an assay using a circRNA microarray to profile the expression of circRNAs. We demonstrated that the expression of 189 circRNAs was significantly different in the M1 compared with that found in the M2 macrophages. Among these, 62 circRNAs were upregulated, while 127 circRNAs were downregulated.
Based on the microarray analysis, high levels of circRNA003780, circRNA-010056 and circRNA-010231 in M1 cells and circRNA-003424, circRNA-013630, circRNA-001489 and circRNA-018127 in M2 cells with fold-change $>5$ were selected and validated by RT-qPCR to confirm the results of the microarray analysis. Although, there were some discrepancies in the results of the microarray and the RT-qPCR analyses, the microarray provided a rapid method for identifying a large number of differentially expressed circRNAs in M1 macrophages which could then be confirmed by RT-qPCR.

Recent evidence suggests that circRNAs play a crucial role in fine-tuning the level of miRNA-mediated regulation of gene expression by sequestering miRNAs. The interac- 
tion of circRNAs with disease-associated miRNAs indicates that circRNAs are important for disease regulation (26). For example, the circRNA ciRS-7 contains multiple tandem miRNA-7 binding sites, thereby acting as an endogenous miRNA 'sponge' to adsorb, and hence quench normal miRNA-7 function (27). Thus, we annotated the circRNA/miRNA interaction for the differentially expressed circRNAs and performed a detailed annotation for representing the binding sites of circRNA-010231 and the conserved MREs with good scores with miR-6950-5p, miR-19-2-5p, miR-141-5p, miR-1964-5p and miR-145a-3p, respectively. However, few circRNA/miRNA interactions have been experimentally validated. Therefore, the functional effect of their interaction will be the focus of our future research.

In conclusion, the present study investigated the global expression patterns of circRNAs in macrophage activation and contributes to the understanding of the role of circRNAs in macrophage polarization under different stimulating conditions. The illustration from our database is an important starting point for understanding the role of circRNAs in macrophage function and their potential effect on disease pathology.

\section{Acknowledgements}

The present study was supported by grants from the National Natural Science Foundation of China (Nos. 81300172, 81301497 and 81472017) and the Natural Science Foundation of Anhui Province (Nos. 1308085QH137 and 1408085QH148).

\section{References}

1. Gordon S and Martinez FO: Alternative activation of macrophages: Mechanism and functions. Immunity 32: 593-604, 2010.

2. Mosser DM: The many faces of macrophage activation. J Leukoc Biol 73: 209-212, 2003

3. Biswas SK and Mantovani A: Macrophage plasticity and interaction with lymphocyte subsets: Cancer as a paradigm. Nat Immunol 11: 889-896, 2010.

4. Mantovani A, Sozzani S, Locati M, Allavena P and Sica A: Macrophage polarization: Tumor-associated macrophages as a paradigm for polarized M2 mononuclear phagocytes. Trends Immunol 23: 549-555, 2002.

5. Chen T, Huang Z, Wang L, Wang Y, Wu F, Meng S and Wang C: MicroRNA-125a-5p partly regulates the inflammatory response, lipid uptake, and ORP9 expression in oxLDL-stimulated monocyte/macrophages. Cardiovasc Res 83: 131-139, 2009.

6. Cheng Y, Kuang W, Hao Y, Zhang D, Lei M, Du L, Jiao H, Zhang $X$ and Wang F: Downregulation of miR-27a* and miR-532-5p and upregulation of miR-146a and miR-155 in LPS-induced RAW264.7 macrophage cells. Inflammation 35: 1308-1313, 2012.

7. Coley W, Van Duyne R, Carpio L, Guendel I, Kehn-Hall K, Chevalier S, Narayanan A, Luu T, Lee N, Klase Z, et al: Absence of DICER in monocytes and its regulation by HIV-1. J Biol Chem 285: 31930-31943, 2010.
8. Dalton DK, Pitts-Meek S, Keshav S, Figari IS, Bradley A and Stewart TA: Multiple defects of immune cell function in mice with disrupted interferon-gamma genes. Science 259: 1739-1742, 1993.

9. Forrest AR, Kanamori-Katayama M, Tomaru Y, Lassmann T, Ninomiya N, Takahashi Y, de Hoon MJ, Kubosaki A, Kaiho A, Suzuki M, et al: Induction of microRNAs, mir-155, mir-222, mir-424 and mir-503, promotes monocytic differentiation through combinatorial regulation. Leukemia 24: 460-466, 2010.

10. Goerdt $S$ and Orfanos CE: Other functions, other genes: Alternative activation of antigen-presenting cells. Immunity 10 : 137-142, 1999.

11. Gordon S: Alternative activation of macrophages. Nat Rev Immunol 3: 23-35, 2003.

12. Cai X, Yin Y, Li N, Zhu D, Zhang J, Zhang CY and Zen K: Re-polarization of tumor-associated macrophages to pro-inflammatory M1 macrophages by microRNA-155. J Mol Cell Biol 4: 341-343, 2012.

13. Memczak S, Jens M, Elefsinioti A, Torti F, Krueger J, Rybak A, Maier L, Mackowiak SD, Gregersen LH, Munschauer M, et al: Circular RNAs are a large class of animal RNAs with regulatory potency. Nature 495: 333-338, 2013.

14. Ebert MS, Neilson JR and Sharp PA: MicroRNA sponges: Competitive inhibitors of small RNAs in mammalian cells. Nat Methods 4: 721-726, 2007.

15. Jeck WR, Sorrentino JA, Wang K, Slevin MK, Burd CE, Liu J, Marzluff WF and Sharpless NE: Circular RNAs are abundant, conserved, and associated with ALU repeats. RNA 19: 141-157, 2013.

16. Jeck WR and Sharpless NE: Detecting and characterizing circular RNAs. Nat Biotechnol 32: 453-461, 2014.

17. Hansen TB, Jensen TI, Clausen BH, Bramsen JB, Finsen B, Damgaard CK and Kjems J: Natural RNA circles function as efficient microRNA sponges. Nature 495: 384-388, 2013.

18. Zhang Y, Zhang XO, Chen T, Xiang JF, Yin QF, Xing YH, Zhu S, Yang $L$ and Chen LL: Circular intronic long noncoding RNAs. Mol Cell 51: 792-806, 2013

19. Lasda E and Parker R: Circular RNAs: Diversity of form and function. RNA 20: 1829-1842, 2014.

20. Salzman J, Chen RE, Olsen MN, Wang PL and Brown PO: Cell-type specific features of circular RNA expression. PLoS Genet 9: e1003777, 2013.

21. Enright AJ, John B, Gaul U, Tuschl T, Sander C and Marks DS: MicroRNA targets in Drosophila. Genome Biol 5: R1, 2003.

22. Pasquinelli AE: MicroRNAs and their targets: Recognition, regulation and an emerging reciprocal relationship. Nat Rev Genet 13: 271-282, 2012.

23. Zhang Y, Zhang M, Zhong M, Suo Q and Lv K: Expression profiles of miRNAs in polarized macrophages. Int J Mol Med 31: 797-802, 2013

24. Rückerl D, Jenkins SJ, Laqtom NN, Gallagher IJ, Sutherland TE, Duncan S, Buck AH and Allen JE: Induction of IL-4R $\alpha$ dependent microRNAs identifies PI3K/Akt signaling as essential for IL-4-driven murine macrophage proliferation in vivo. Blood 120: 2307-2316, 2012.

25. Chaudhuri AA, So AY, Sinha N, Gibson WS, Taganov KD, O'Connell RM and Baltimore D: MicroRNA-125b potentiates macrophage activation. J Immunol 187: 5062-5068, 2011.

26. Ghosal S, Das S, Sen R, Basak P and Chakrabarti J: Circ2Traits: A comprehensive database for circular RNA potentially associated with disease and traits. Front Genet 4: 283, 2013.

27. Lukiw WJ: Circular RNA (circRNA) in Alzheimer's disease (AD). Front Genet 4: 307, 2013. 